\title{
Defining a role for exercise training in the management of asthma
}

\author{
Anna T. Freeman ${ }^{1,2}$, Karl J. Staples $\mathbb{1}^{1,2,3}$ and Tom M.A. Wilkinson ${ }^{1,2,3}$ \\ Affiliations: ${ }^{1}$ Clinical and Experimental Sciences, University of Southampton Faculty of Medicine, \\ Southampton, UK. ${ }^{2}$ NIHR Southampton Biomedical Research Centre, University Hospital Southampton NHS \\ Foundation Trust, Southampton, UK. ${ }^{3}$ Wessex Investigational Sciences Hub, University of Southampton Faculty \\ of Medicine, Southampton General Hospital, Southampton, UK.
}

Correspondence: Anna T. Freeman, Mailpoint 810, Level F, South Block, Southampton General Hospital, Southampton S016 6YD, UK. E-mail: A.Freemandasouthampton.ac.uk

@ERSpublications

Asthma remains prevalent and many patients are symptomatic despite optimised treatment regimes. This review explores the role of exercise as an immunomodulatory treatment in asthma, and the barriers to this. http://bit.ly/2sE6IE8

Cite this article as: Freeman AT, Staples KJ, Wilkinson TMA. Defining a role for exercise training in the management of asthma. Eur Respir Rev 2020; 29: 190106 [https://doi.org/10.1183/16000617.0106-2019].

ABSTRACT The prevalence of asthma remains high worldwide, with increasing awareness of the morbidity and mortality from asthma in low-income countries. In the UK, despite the development of biological treatments, many patients remain suboptimally controlled, and mortality rates have been static for decades. Therefore, new approaches are needed to treat asthma that are scalable at minimal cost. Exercise immunology is an expanding field, and there is growing evidence that exercise can modulate inflammatory and immune processes in asthma. Whilst exercise is encouraged in current treatment guidelines, there are no specific recommendations as to the intensity, frequency or duration of exercise exposure. Despite national and international guidance to increase exercise, patients with asthma are less likely to engage in physical activity. This review explores the disease modifying benefit of exercise in asthma. We also review the domains in which exercise exerts positive clinical effects in asthma, including the effects of exercise on symptom scores, quality of life, psychosocial health, and in the obese asthma phenotype. Finally, we review the barriers to exercise in asthma, given the benefits it confers. A better understanding of the mechanisms through which exercise exerts its positive effects in asthma may provide more accurate prescription of exercise training programmes as part of broader asthma management, with the potential of identification of new drug targets.

\section{Introduction}

The burden of respiratory disease is increasing, with asthma affecting over 300 million people worldwide. In children, asthma is the most common chronic disease [1], and amongst the highest global ranking for disability-adjusted life-years [2]. Historically, the prevalence of asthma in low-income countries was thought to be minimal, but phase three of the International Study of Asthma and Allergies in Childhood suggests otherwise, with the prevalence of asthma, rhinoconjunctivitis and eczema demonstrated to be high in nonaffluent areas with low socioeconomic conditions [3]. Similar trends for prevalence have been noted in adults, with a recent review concluding that further increases worldwide are likely. Despite development of biological treatments, many adult asthma patients remain suboptimally controlled, with frequent exacerbations, increased symptom burden and associated healthcare costs [4], with low-income countries demonstrating higher levels of poor control amongst both children and adults [5]. Biological treatments for children are increasingly available but current understanding of which children are likely to benefit from these is limited, as the majority of trial data exists in adults [6]. It is probable that, as with adults, there will

Provenance: Submitted article, peer reviewed

Received: 13 Aug 2019 | Accepted after revision: 18 Dec 2019

Copyright $\odot$ ERS 2020. This article is open access and distributed under the terms of the Creative Commons Attribution Non-Commercial Licence 4.0. 
be groups of patients for whom there is no suitable biological target or response to biological treatment is limited. Therefore, new approaches are needed to treat asthma that are scalable at minimal cost. Whilst exercise is encouraged in current national and international treatment guidelines for both children and adults, there are no specific recommendations as to the intensity, frequency or duration of exercise exposure [7]. Despite guidance to increase levels of exercise, patients with asthma are less likely to engage in physical activity [8]. Exercise, as pulmonary rehabilitation, has an established role in treatment of respiratory disease, but is not routinely employed in the management of complex asthma [9]. Part of this may be that the majority of asthma patients are less functionally disabled than the patients with COPD who engage with pulmonary rehabilitation, and therefore both clinicians and patients fail to see the benefit. There may also be the concern of exercise precipitating an exacerbation in patients with suboptimal control. There is, however, suggestion that the worse the asthma control, the greater improvement is demonstrated with pulmonary rehabilitation [10], refuting this concern. Emerging data suggest that exercise as a therapeutic intervention may modulate the immune and inflammatory basis for respiratory disease, and offer clinical benefit beyond functional capacity improvement through disease modification [11, 12], suggesting a potential cost-effective benefit to a wide range of suboptimally controlled asthma patients.

Exercise immunology is an expanding field, and recently, studies have demonstrated the effects of exercise on inflammation and immunity [13]. Whilst the immunosuppressive effects of extreme exercise in athletes has been well described, a recent review suggests the interaction between exercise and immune function may be more complex than traditionally thought [14]. Within athletes with asthma, there is the further consideration of potential worsening of symptoms due to exercise-induced bronchoconstriction and exposure to environmental factors such as cold air, chloramines in pool environments and allergens as exacerbators of disease, and this needs to be considered in the context of an exercise intervention as a nonpharmacological treatment for asthma. However, here, we focus instead on the role of more moderate exercise interventions in previously untrained populations. The use of exercise intervention as an anti-inflammatory treatment in chronic diseases is a more recently emerging research area and, within asthma, human mechanistic studies are lacking.

The Global Initiative for Asthma defines asthma as "a heterogenous disease, usually characterised by airway inflammation" with symptoms of "wheeze, shortness of breath, chest tightness and cough that vary over time and in intensity, together with variable expiratory flow limitation" [15]. It is thought that this chronic inflammatory state drives the symptom burden of asthma, with oxidative stress implicated in its pathogenesis [16]. There is growing evidence that exercise modulates this redox balance [17], as well as the inflammatory and immune processes in asthma [11, 12]. Beyond this generalised description, it is increasingly recognised that asthma encompasses several phenotypes and endotypes [18], with significant differences in disease patterns and treatment responsiveness between patients. HALDAR et al. [18] identified four key clusters in their secondary care cohort; 1) early-onset atopic asthma; 2) female-predominant obese noneosinophilic asthma; 3) early-onset symptom-predominant asthma with minimal eosinophilic disease; and 4) male-predominant later-onset eosinophilic disease with fewer symptoms. Unlike targeted biological therapies, exercise as an intervention has the potential to improve control across a broad range of disease endotypes, and exercise intervention studies to date tend not to have investigated specific phenotypes or endotypes in isolation. Whilst biological therapies are emerging as a key treatment for asthma, many patients do not demonstrate the appropriate biological marker, or remain suboptimally controlled in spite of biological treatment $[19,20]$, hence new treatment targets are required. Understanding the mechanisms through which exercise improves asthma control and inflammation will help establish exercise as a therapeutic intervention and identify patient endotypes most likely to benefit. Better mechanistic understanding may also offer the potential to identify new therapeutic targets for medication.

This review explores anti-inflammatory effects of exercise in asthma, and the potential mechanisms behind this. We review the clinical domains in which exercise exerts positive effects on symptoms. Finally, we explore. the barriers to exercise in asthma.

\section{Effects of exercise on inflammation and immunity in asthma}

Whilst exercise should benefit asthma patients in terms of their symptom burden regardless of phenotype, it is in allergic and eosinophilic disease that there is most interest in exercise as a disease modifier. Allergic and eosinophilic asthma involves chronic inflammation and airway remodelling [21], with T-helper cells (Th)2 dominating the T-cell response [22], resulting in an increase of airway eosinophils, CD4 ${ }^{+} \mathrm{T}$-cells and mast cells [21]. Th2 cytokines interleukin (IL)-4, IL-5 and IL-13 initiate and propagate inflammation, with eventual airway remodelling, hyperresponsiveness and obstruction.

Animal studies

Much of the mechanistic work in this context has been undertaken in mice, using ovalbumin (OVA)-sensitised mice as a model for allergic asthma (table 1). This model demonstrated decreased 
TABLE 1 Summary of findings from animal studies

First author [ref.]

PASTVA [23]

Mouse model

6-9 female BALB/cJ mice; sedentary and nonsensitised, sedentary and OVA-sensitised, exercised and nonsensitised, and exercised and OVA-sensitised

Pastva [24]

VIEIRA [25]

HEWITT [26]

SILVA [21]

LOWDER [27]

VIEIRA [28]

SILVA [29]
Female BALB/cJ mice split into six groups; sedentary and nonsensitised sedentary and OVA-sensitised with placebo; sedentary and OVA-sensitised with RU486 treatment; exercised and nonsensitised; exercised and

OVA-sensitised with placebo; and exercised and OVA-sensitised with RU486 treatment

8 per group OVA-sensitised male BALB/ $c$ mice versus sensitised and nonsensitised controls

Female BALB/cJ mice

7 male BALB/c mice in four groups; control; AT; OVA; and OVA+AT

Female C.Cg-Foxp3tm2Tch/J reporter (Foxp3 $3^{+}$reporter) mice bred on a BALB/cJ background

8 per group ovalbumin sensitised male $\mathrm{BALB} / \mathrm{c}$ mice versus sensitised and nonsensitised controls

8 male BALB/c mice in four groups; control (nontrained and nonsensitised); $\mathrm{AC}$ laerobic conditioning and nonsensitised); OVA (nontrained and OVA-sensitised); and OVA+AC (aerobic conditioning and OVA-sensitised)

Intervention

Significant outcomes

Thrice weekly 45-min sessions for 4 weeks of aerobic exercise at $50 \% V_{\text {, }_{2} \text { peak }}$

Thrice weekly 45-min sessions for 4 weeks of aerobic exercise at $50 \% V_{\mathrm{O}_{2} \text { peak }}^{\prime}$

Low-intensity training at $50 \%$ maximal speed and moderate intensity at $75 \%$ maximal speed for 60 min $5 \times$ week for 28 days

Single 45-min bout of aerobic exercise at $50-75 \% V^{\prime}{ }_{O_{2} \text { peak }}$

$5 \times 60$-min sessions for 4 weeks at $50 \% V^{\prime}{ }_{\mathrm{O}_{2} \text { peak }}$

45 mins $3 \times$ week for 4 weeks at $50-75 \%$ maximum $\mathrm{O}_{2}$ consumption

Low-intensity training $60 \mathrm{~min}$ $5 \times$ week for 28 days

60 min for 5 days per week for 8 weeks at $50 \% V^{\prime}{ }_{O_{2} \text { peak }}$ prior to and during OVA sensitisation
Reduced BAL IL-4, IL-5 and OVA-specific $\lg \mathrm{E}$

Reduced chemokine production Reduced lung cellular infiltrate, mucus production and epithelial hypertrophy decreased both the phosphorylation of $\mathrm{I} \kappa \mathrm{B}-\alpha$ and translocation of the NF- $\kappa \mathrm{B}$ subunit $\mathrm{p} 65$

RU486 blocked the exercise-induced reductions in cellular infiltration of the airways $\mathrm{KC}$ and soluble VCAM- 1 protein

levels in the BAL fluid and NF-KB translocation and DNA binding within the lung to levels similar to those observed in sedentary OVA-sensitised mice

Reduced airway wall and BAL eosinophils in both exercised groups

Decreased peri-bronchial density of cells positive for IL-4 and IL-5, and increased IL-10

Reduced airway wall collagen and elastin fibres in airways

Normalisation of bronchoconstriction index

Decreased leukocyte infiltration, including eosinophils

Decreased phosphorylation of the NF- $\mathrm{KB}$ p65 subunit

Decreased IL-5, IL-3 and PGE 2

Increase of $\lg E$ and $\lg G$

Reduction of eosinophils, $\mathrm{CD}^{+}, \mathrm{CD}^{+}, \mathrm{IL}-4$,

IL-5, IL-13, NF- $\mathrm{B}$, airway remodelling,

mucus synthesis, smooth muscle thickness and tissue resistance and elastance

Increase in IL-10 and IL-1ra independently of Foxp3

Reduced BAL macrophages, eosinophils and lymphocytes

Enhanced the suppression function of $\mathrm{CD}^{+}{ }^{+} \mathrm{CD} 25^{+} \mathrm{Foxp}^{+} \mathrm{T}$-reg cells

Reduced total cells and eosinophils in BAL fluid

Reduced percentage of goblet cells in airway walls

Reduced epithelial expression of IL-4,5,

IL-13 and increased IL-10

Reduced inducible nitric oxide synthase, and other markers of oxidative and nitrosative stress

Reduced NF-kB and P2X7 receptor

Inhibition of OVA-induced migration of eosinophils and lymphocytes to the airways Reduced $\lg E$ and $\lg \mathrm{G} 1$ titres Inhibition of Th2 cytokines 
TABLE 1 Continued

First author [ref.]

DUGGER [30]

BRUgGemann [31]

8 male Swiss mice per group; control swimming OVA-sensitised and

OVA-sensitised+swimming

30-min high-intensity swimming for 3 weeks

Alberca-Custodio [32] 16 per group; control, exercise, OVA and OVA+exercise groups

FERNANDES [33]

$10 \mathrm{BALB} / \mathrm{c}$ mice in four groups; control, exercise, OVA, and OVA exercise group

Intervention

$45 \mathrm{~min}$ thrice weekly for 4 weeks at $13.5 \mathrm{~m}$ per $\mathrm{min}$

OVA-sensitised; exercised

OVA-sensitised; sedentary

nonsensitised

OVAtexercise groups

$60 \min 5 \times$ week for 4 weeks

5 weeks exercise for $60 \mathrm{~min}$ $5 \times$ week at $50 \% \mathrm{~V}_{\mathrm{O}_{2} \text { peak }}$

Significant outcomes

Surface expression levels of lung-homing

chemokine receptors were comparable across groups

Lung-derived Th cells from exercised

OVA-sensitised mice exhibited decreased migratory function versus controls; Th cells from exercised mice are less responsive to lung-homing chemokines

Decreased OVA-increased total IgE, IL-1, IL-4, IL-5 and IL-6 levels, total cells, lymphocytes and eosinophils in BAL fluid Increased IL-10 and glutathione levels Increased glutathione peroxidase and catalase in the swimming-only group

Decreased eosinophils neutrophils lymphocytes and macrophages in BAL Decreased eosinophils

lymphocytes and macrophages in airway walls

Reduced collagen elastic fibres, mucus production, and smooth muscle thickness Reduced IL-5, IL-13, cysLT and $\mathrm{LTB}_{4}$ in BAL

Reduced 5-LO, LTA $_{4 \mathrm{H}}$, cys LT 1 receptor, Cys $\mathrm{LT}_{2}$ receptor, $\mathrm{LTC}+$ synthase and $\mathrm{BLT} 2$ expression by peri-bronchial leukocytes and airway epithelium

Reduced AHR to methacholine

Increased IL-10 and TGF- $\beta$ Increased recruitment of $\mathrm{M} 2$ in the lungs, influx and activation of Tregs and $\mathrm{CD}^{+}$ and $\mathrm{CD} 8^{+}$lymphocytes

Decreased proinflammatory common dendritic cells' expression of co-stimulatory molecules Increased anti-inflammatory ICOSL in plasmacytoid dendritic cells

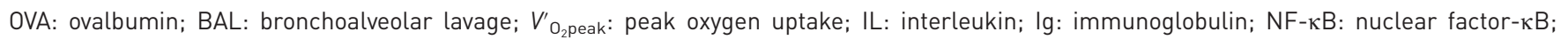
VCAM: vascular cell adhesion molecule; $\mathrm{PGE}_{2}$ : prostaglandin $\mathrm{E}_{2}$; AT: anaerobic threshold; Treg: regulatory T-cell; Th: T-helper; cysLT: cysteinyl leukotriene; $\mathrm{LTB}_{4}$ : leukotriene $\mathrm{B}_{4}$; LTC: leukotriene synthase; LTA ${ }_{4 \mathrm{H}}$ : leukotriene A4 hydrolase; AHR: airway hyperresponsiveness; TGF- $\beta$ : transforming growth factor $\beta$; ICOSL: inducible T-cell co-stimulator ligand.

leukocyte infiltration, cytokine production (IL-4, IL-5, IL-13), adhesion molecule expression, and structural remodelling within the lungs following a 4-week period of moderate intensity exercise [21, 23, 25]. In addition, VIEIRA et al. [25] and SiLVA et al. [21] demonstrated a reduction in activation of the inflammatory transcription factor nuclear factor $-\kappa \mathrm{B}(\mathrm{NF}-\kappa \mathrm{B})$ with aerobic training. Exercise reduced airway remodelling, mucus synthesis, smooth muscle thickness and tissue resistance and elastance, and increased IL-10 and IL-1ra. SiLva et al. [21] found this change occurred independently of regulatory T-cell (Treg) cell activity (FoxP3), whereas LowDER et al. [27] found that exercise increased levels of FoxP3 ${ }^{+}$cells in the lungs and mediastinal lymph nodes of OVA-sensitised mice.

A single bout of moderate intensity exercise in OVA-sensitised mice causes similar decreases in leukocyte infiltration, including eosinophils, within the lungs [26]. There was decreased phosphorylation of the NF- $\kappa B$ p65 subunit and reduced IL-5, IL-13 and prostaglandin $E_{2}$. There was attenuation of the chemokines KC, RANTES and monocyte chemoattractant protein (MCP)-1. However, a single bout of exercise had no effect on airway hyperresponsiveness, epithelial cell hypertrophy, mucus production, or airway wall thickening. 
When aerobic conditioning was performed before and during OVA sensitisation of mice, rather than after, a similar reduction in inflammation was observed. Exercise reduced the OVA-induced migration of eosinophils and lymphocytes into the airways, and reduced expression of Th2 cytokines, as well as expression of intracellular adhesion molecule (ICAM)-1, vascular cellular adhesion protein (VCAM)-1, RANTES, transforming growth factor (TGF)- $\beta$ and vascular endothelial growth factor (VEGF). Airway remodelling and production of allergen-specific immunoglobulin (Ig)E and IgG-1 were reduced [29], suggesting exercise may be protective to subsequent inflammatory insults.

The mechanisms of how exercise exerts its anti-inflammatory effects in this simplified mouse model of asthma remain unclear, although reduced NF- $\mathrm{KB}$ activation [23], increases in glucocorticoid receptor expression [24], FoxP3 increases [27], T-cell trafficking in response to an allergen challenge [30] with increased lung recruitment of Tregs and macrophages [33], and increased IL-10 and IL-1ra have been proposed $[25,21]$, with IL-10 increases recently directly linked with increases in M2 macrophages in the lung tissue [33].

\section{Potential mechanisms to explain the anti-inflammatory effect of exercise in animal models}

In search of a mechanistic link within this animal model, AlBERCA-Custodio et al. [32], looked specifically at whether moderate intensity exercise modulates the leukotriene pathway in OVA mice. They found that moderate aerobic exercise reduced eosinophils in bronchoalveolar lavage (BAL) and airway walls, and reduced IL-5 and IL-13 in BAL, which was associated with reduced total cysteinyl leukotrienes (cysLTs) in the BAL and airway epithelial cells of exercised OVA mice, as well as reduced airway hyperresponsiveness. It is known that cysLTs activate NF- $\mathrm{KB}$. In OVA-sensitised mice treated with a cysLT receptor antagonist [34], corticosteroids were shown to inhibit cysLTs, which may contribute to the of the mechanism through which they exert their anti-inflammatory effects. As NF- $\kappa B$ is activated by cysLTs, this provides support for the role of NF- $\mathrm{KB}$ and cysLTs as a mechanism for reduced inflammation in exercise.

PASTVA et al. [24] treated exercised OVA mice with the glucocorticoid receptor antagonist RU486, and with this, exercise-induced changes in airway infiltration and BAL levels of KC and VCAM-1 were reduced to levels of sedentary mice, as were NF- $\mathrm{BB}$ translocation and DNA binding within the lung, and exercise-induced increases in glucocorticoid receptor nuclear translocation. Silva et al. [35] looked at the timescales of these changes and found that the OVA-induced reduction in glucocorticoid receptor expression was attenuated at day 3 of exercise training. Exercise training reversed the OVA-induced increase in the expression of NF- $\mathrm{KB}$ at 7 days, with increased expression of IL-10 and IL-1ra, and no change in the expression of FoxP3. Eosinophil migration into the airways, and expression of ICAM-1 and VCAM-1 in the exercised OVA group reduced at this time-point, as did IL-4, IL-5, exotoxin and RANTES. OVA-induced levels of vascular endothelial growth factor and transforming growth factor- $\beta$ also reduced at day 7. The timescales demonstrated in this study suggest that modifications in the status of the glucocorticoid receptor may initiate the anti-inflammatory changes induced by exercise.

Alterations in airway epithelium are important in inflammation and airway remodelling in asthma. VIEIRA et al. [33] investigated the effects of aerobic exercise on airway epithelial cells in OVA-sensitised mice and showed that low-intensity aerobic exercise reduced oxidative and nitrosative stress. There was reduced epithelial expression of NF- $\mathrm{KB}$ and P2X7R (a plasma membrane receptor involved in control of proinflammatory cytokine expression) and increased expression of epithelial IL-10. In a similar mouse model, high-intensity swimming resulting in increased glutathione with attenuation of pulmonary allergic inflammation, with the authors suggesting the effects of swimming were partly mediated through reduced oxidative stress and increased IL-10 production [31].

\section{Effect of exercise on inflammation in children}

Intervention studies of exercise as a disease-modulating treatment for children with asthma are small, scarce and tend to focus on clinical improvements as primary outcomes (table 2). Changes in markers of inflammation are recorded as a secondary outcome, but without mechanistic work to investigate how these changes are affected.

In atopic, asthmatic, school-aged children, a 12-week moderate intensity exercise programme reduced mite-specific IgE, with no change in exhaled nitric oxide fraction $\left(F_{\mathrm{eNO}}\right)$, blood eosinophil levels or serum C-reactive protein [37]. High-intensity interval training in children demonstrates an improvement in body mass index (BMI) and aerobic fitness but without improving inflammation as assessed by $F_{\mathrm{eNO}}$ [41]. The most mechanistic work to date was undertaken in adolescents, and demonstrated that exercise training reduced glucocorticoid receptor expression in leukocytes and monocytes [38]. The authors postulated that exercise training reduced the stress response overall, resulting in a reduction in glucocorticoid receptor expression in several lymphocyte subsets after an acute exercise challenge and sustained after an exercise training intervention. An 8-week exercise intervention improved lung function in children compared to 
TABLE 2 Summary of findings from studies investigating exercise interventions in children with asthma

\begin{tabular}{|c|c|c|c|}
\hline First author [ref.] & Patient demographics & Intervention & Significant outcomes \\
\hline MoREIRA [37] & $\begin{array}{c}17 \text { versus } 17 \text { control } \\
\text { Children aged } 12.7 \\
\pm 3.5 \text { years } \\
\text { Atopic } \\
\text { On maintenance OCS }\end{array}$ & $\begin{array}{l}\text { 50-min twice-weekly training session for } \\
3 \text { months } \\
30-35 \text { min submaximal training }\end{array}$ & $\begin{array}{c}\text { Improved } \mathrm{pAQLQ} \\
\text { Reduced total } \lg \mathrm{E} \text { and mite-specific } \lg \mathrm{E}\end{array}$ \\
\hline ONUR [17] & $\begin{array}{l}15 \text { with } 15 \text { asthmatic } \\
\text { controls and } 15 \text { healthy } \\
\text { controls }\end{array}$ & 8-week exercise training intervention & $\begin{array}{l}\text { Decreased oxidant stress markers } \\
\text { Increased antioxidant enzyme activity } \\
\text { Improved lung function }\end{array}$ \\
\hline WILLEBOORDSE [34] & $\begin{array}{l}43 \text { with } 44 \text { control } \\
\text { Median age } 12.3 \text { years } \\
\text { Median BMI } 25 ; 53 \% \text { of } \\
\text { group obese }\end{array}$ & $\begin{array}{c}60 \text { min } 2 \times \text { week at } 60-75 \% \text { maximal } \\
\text { heart rate for } 6 \text { months }\end{array}$ & $\begin{array}{l}\text { Improved asthma control and quality of life, } \\
\text { reduced weight and improved FVC }\end{array}$ \\
\hline Lu [38] & $\begin{array}{l}12 \text { versus } 14 \text { healthy } \\
\text { adolescents } \\
\text { Mean BMI } 22\end{array}$ & $\begin{array}{l}\text { Acute exercise challenge followed by } \\
60 \text { min thrice weekly for } 8 \text { weeks }\end{array}$ & $\begin{array}{l}\text { Reduced glucocorticoid receptor expression in } \\
\text { several lymphocyte subsets after an acute } \\
\text { challenge and } 8 \text { weeks of training }\end{array}$ \\
\hline Abdelbasset [39] & $\begin{array}{c}19 \text { with } 19 \text { control } \\
\text { Children aged } 8-12 \text { years } \\
\text { with moderate persistent } \\
\text { asthma }\end{array}$ & $\begin{array}{c}40 \text { min thrice weekly training sessions at } \\
50-70 \% \text { maximum heart rate for } \\
10 \text { weeks }\end{array}$ & $\begin{array}{l}\text { Improved } \mathrm{FEV}_{1} \text { and } \mathrm{FVC} \\
\text { Improved } \mathrm{V}^{\prime} \mathrm{O}_{2} \text { peak } \\
\text { Improved } 6 \mathrm{MWT}\end{array}$ \\
\hline LUCAS [40] & $\begin{array}{c}86 \text { asthma, } 142 \\
\text { nonasthmatic obese } \\
\text { Mean BMI percentile } 98.14 \\
\text { Mean age } 11.26 \text { years }\end{array}$ & $\begin{array}{c}40 \text { min physiologist-designed exercise } \\
\text { weekly }\end{array}$ & Reduced weight and improved $V^{\prime}{ }_{O_{2} \text { peak }}$ \\
\hline
\end{tabular}

AT: anaerobic threshold; $V^{\prime}{ }_{O_{2} \text { peak: }}$ peak oxygen uptake; OCS: oral corticosteroids; pAQLQ: paediatric Asthma Quality of Life Questionnaire; Ig: immunoglobulin; BMI: body mass index; FVC: forced vital capacity; FEV 1 : forced expiratory volume in $1 \mathrm{~s}$; 6MWT: 6-min walk test.

pharmacological management only, along with an improvement in levels of oxidative stress [17]. Otherwise, exercise intervention studies in children have tended to focus more on symptom control and quality of life as outcomes.

\section{Effect of exercise on inflammation in adults}

Exercise intervention studies in adults similarly focus on clinical outcome with inflammatory changes as secondary outcomes (table 3). An exercise intervention study in adults examined moderate-to-severe adult asthma patients who completed a twice-weekly exercise programme of 3 months' duration, with a control group of matched patients undergoing a breathing training programme.

Here, induced sputum eosinophil counts fell in the training group after exercise, as did $F_{\text {eNO}}$, with greatest reductions seen with highest baseline levels [44]. Asthma symptoms also reduced in the training group [44]. Reduction in sputum eosinophils was also demonstrated in an exercise and dietary intervention in obese asthma patients [46]. Others have shown that aerobic training at moderate intensity reduces bronchial hyperreactivity, with reduced IL-6 and MCP-1 [12]. Quality of life, as measured by the Asthma Quality of Life Questionnaire (AQLQ) results and asthma exacerbation rates also improved. The effects of exercise and weight loss were explored in a randomised control trial of obese asthmatic adults; the combined programme demonstrated an improvement in clinical symptoms and aerobic capacity, accompanied by weight loss. There was also reduction in $F_{\mathrm{eNO}}$, CCL2, IL-4, tumour necrosis factor- $\alpha$ and leptin, with increased levels of vitamin $25(\mathrm{OH}) \mathrm{D}, \mathrm{IL}-10$ and adiponectin [11]. However, the exercised group showed a significantly larger reduction in BMI, and therefore it is impossible to exclude confounding from reduced obesity-driven systemic inflammation. High-intensity intermittent exercise training without strength training has been looked at in adult asthmatic patients [49], and was shown to be beneficial in terms of symptom scores as assessed by Asthma Control Questionnaire (ACQ) and AQLQ. There were no significant changes in 
TABLE 3 Summary of findings from studies investigating exercise interventions in adults with asthma

Study Patient demographics Intervention Significant outcomes

FARID [42]

Mendes [43]

Mendes [44]

TURNER [45]

ScotT [46]

Franca-Pinto [12]

FREITAS [47]

TURK [48]

CANDEmir [9]

Toennesen [49]

FREITAS [11]
18 versus 18 controls

Mean age 29 years

Exercise-induced asthma

Atopic

44 versus 45 controls

Median age 39 years

Median BMI 25.2

Moderate or severe persistent asthma

34 versus 34 controls

Median age 34 years

Median BMI 25.8

Moderate or severe persistent asthma

19 versus 15 controls

Mean age 71 years

Mean BMI 26.8

Moderate-to-severe asthma with

fixed airflow obstruction

10 exercise versus 15 dietary intervention versus 13 diet and exercise

Mean BMI 33.7

$$
\begin{gathered}
22 \text { versus } 21 \text { controls } \\
\text { Mean age } 40 \text { years } \\
\text { Mean BMI } 26.5 \\
91 \% \text { atopy } \\
\text { Mean maintenance ICS } 909 \\
\text { Moderate-severe asthma }
\end{gathered}
$$

28 versus 27 controls

Mean age 45.9 years

Mean BMI 38.1

Obese participants

44 versus 30 controls

Obese mean BMI 44.75

$$
\begin{gathered}
35 \text { patients } \\
\text { Mean age } 45 \text { years }
\end{gathered}
$$

29 exercise; 29 diet and exercise, 33 diet, 34 control

Mean age 38.2-43.7 years

Mean BMI 24.9-26.1

$72-85 \%$ atopy

$59-76 \%$ ICS

28 versus 27 controls

Mean age 45.9 years

Mean BMI 38.1

Obese participants
$3 \times$ weekly 20 min aerobic exercise for 8 weeks

$2 \times 30$-min training session on indoor treadmill for 3 months

$$
60-80 \% V^{\prime}{ }_{O_{2}} \max
$$

$2 \times 30$-min training session on indoor treadmill for $3 / 12$

$60-80 \% V^{\prime}{ }_{\mathrm{O}_{2} \max }$

$1 \times$ weekly 20 min walking at $80 \%$ of average walking speed on $6 \mathrm{MWT}$ circuit training based on Borg RPE scale

Personal training 60 min per week and visit gym $3 \times$ week for 12 weeks

2xweekly 35 min (25-min training, 5-min warm up/cool down)

"vigorous" aerobic training programme on an indoor treadmill for 3 months

2×weekly constant WR aerobic training $50-75 \% V^{\prime}{ }_{\mathrm{O}_{2} \text { peak }}$ and resistance training $60 \% 1 \mathrm{RM}$

Thrice weekly sessions of 40-60 min high-intensity interval training for

$$
12 \text { weeks }
$$

30-min sessions thrice weekly for

8 weeks at $85 \% V^{\prime}{ }_{O_{2} \text { peak }}$ plus

quadriceps resistance training

2-5 repeats of 5-min interval training consisting of: 1) $30 \mathrm{~s}$ at $30 \mathrm{~V}^{\prime} \mathrm{O}_{2}$ max;

2) $20 \mathrm{~s}$ at $\left.60 \% V_{\mathrm{O}_{2} \max } ; 3\right) 10 \mathrm{~s}$ at $90 \%$

$$
V_{\mathrm{O}_{2} \max }^{\prime}
$$

2xweekly constant WR aerobic training $50-75 \% V^{\prime}{ }_{O_{2} \text { peak }}$ and resistance training $60 \% 1 \mathrm{RM}$
Improved $\mathrm{FEV}_{1}, \mathrm{FVC}, \mathrm{PEF}, \mathrm{FEF}_{25-75 \%}$ and MVV

Improved $V^{\prime}{ }_{\mathrm{O}_{2} \max }$ Improved asthma-related QoL Reduced Beck Depression Inventory Reduced State-Trait Anxiety Inventory Scores

Reduced $F_{\text {eNO }}$

Reduced sputum total and eosinophil counts

Increased number of symptom free days Improved $V^{\prime} \mathrm{O}_{2} \max$

Improved AQLQ activity and symptom domains

Improved symptoms with diet and combined

Improved QoL with all three interventions

Reduced sputum eosinophils with exercise Reduced BMI

Improved bronchial hyperreactivity by 1 doubling dose

Reduced serum IL- 6 and MCP-1

Greater proportion of patients with improved depression symptoms

Lower risk of developing OSA Improved sleep quality

Improved asthma control at surgery Improved asthma-related QoL

Improved ACT

Reduced $\mathrm{BMI}$ in obese participants Reduced emergency admissions and hospitalisations

Improved ACQ

Reduced $F_{\text {eNO }}$ Improved $\mathrm{FEV}_{1} / \mathrm{FVC}$

Improved ACQ score

Decreased serum CCL2, IL-4, IL-6, TNF- $\alpha$, leptin

Increased vitamin D, adiponectin, IL-10 
TABLE 3 Continued

Study Patient demographics

Prossegger [50]

\author{
18 exercise, 24 control \\ Mean age 40.6 years \\ HDM sensitisation
}

\begin{abstract}
SAXER [51] 24 versus 24 controls
Low altitude: mean age 47 years, BMI 24

High altitude: mean age 43 years,
\end{abstract} BMI 26

JAAKKOLA [52]

\author{
44 versus 45 controls \\ Mean age 39.7 years \\ Mean BMI 24.97
}

\section{Intervention}

\begin{abstract}
Four 3-5-h guided GPS-monitored hiking/snow-shoe tours with an average altitude difference of $411 \mathrm{~m}$ and $11 \mathrm{~km}$ in distance per day

Four all-day skiing sessions with an average of $42 \mathrm{~km}$ in ski slopes in three different ski regions (2000$2500 \mathrm{ml}$
\end{abstract}

5xweekly 30-45-min guided walks, endurance training, strength training and education for maximum $5 \mathrm{~h}$ per

day for 3 weeks at either $760 \mathrm{~m}$

above sea level or $3100 \mathrm{~m}$ above sea level

$3 \times$ weekly $30-$ min aerobic exercise at $70-80 \%$ maximal HR for 24 weeks

\section{Significant outcomes}

Improved $F_{\text {eNO }}$ Reduced nasal eosinophil count Improvement in allergic symptoms

ACQ in both groups PEF variability in both groups

Improved ACT score

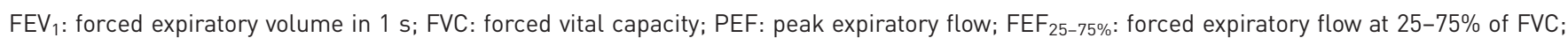
MVV: maximum voluntary ventilation; BMI: body mass index; $V_{\mathrm{O}_{2} \mathrm{max}}$ : maximal oxygen uptake; QoL: quality of life; $F_{\mathrm{eNO}}$ : exhaled nitric oxide fraction; 6MWT: 6-min walk test; RPE: rate perceived exertion; AQLQ: Asthma Quality of Life Questionnaire; ICS: inhaled corticosteroid; IL: interleukin; MCP-1: monocyte chemoattractant protein 1; WR: work rate; $V_{\mathrm{O}_{2} \text { peak }}$ : peak oxygen uptake; RM: repetition maximum; OSA: obstructive sleep apnoea; ACT: Asthma Control Test; ACQ: Asthma Control Questionnaire; TNF: tumour necrosis factor; HDM: house dust mite; HR: heart rate.

anti-inflammatory parameters, and the improvements in fitness and asthma control were not sustained at 1 year [53]. An outdoor walking exercise intervention, which may be more sustainable long term than a gym-based intervention, demonstrated reduction in $F_{\text {eNO }}$ after a single hiking tour, sustained at 10 days. This recreational study demonstrated improvements in allergic symptoms but not in nasal eosinophil cell count at 60 days suggesting some persisting benefit [50]. In an observational context, Del Giacco et al. [54] monitored a professional football team across a season, and found that natural killer cell absolute count and percentage increased in both atopic and nonatopic athletes, and IL- 2 and IL-4 production reduced. The most marked reduction in IL-4 was seen in atopic individuals, suggesting that the immunological mechanisms observed in the short term in murine models and humans are translatable to real-life, longer-term situations, although mechanistic data in asthmatic humans are lacking. Consistently with these early findings, a systematic review of the effect of physical training on airway inflammation in asthma [55] concluded that whilst some findings suggested physical training may reduce airway inflammation in asthma patients, more information was necessary.

\section{Potential mechanisms to explain the anti-inflammatory effect of exercise in children and adults with asthma}

Potential mechanisms include redox modifications of the glucocorticoid receptor, linking in with results from murine studies discussed above [35]. Higher levels of cysteine oxidation of the glucocorticoid receptor have been demonstrated in children with difficult-to-treat asthma, with levels correlating with disease severity and poor control [56]. Greater oxidation of the glucocorticoid receptor in humans promotes post-translational modification that may impair receptor function [56]. Redox regulation and oxidative stress have been implicated in the pathogenesis of asthma [16]. Higher levels of oxidant stress markers have been demonstrated in children with asthma, and a reduction in these markers together with an increase in antioxidant enzyme activity and an improvement in lung function have been shown following an 8-week exercise training intervention [17]. Changes in the interactions between reactive oxygen species in healthy humans undergoing exercise intervention have been demonstrated [57], but this is yet to be investigated in adults with asthma. Additionally, mechanistic involvement of the glucocorticoid receptor does not necessarily fit with the findings of Lu et al. [38] when investigating he effect of exercise on glucocorticoid receptor expression in adolescents.

Given the suggestion of a reduction in inflammation, along with the broader, well-known benefits of increased physical activity, completion of an exercise training intervention would not be an unreasonable 
prerequisite to starting biological treatment and may increase the response rates of these expensive treatments.

\section{Effects of exercise on symptom scores, quality of life and psychosocial morbidity in} asthma in children

It seems exercise confers an anti-inflammatory benefit in patients with asthma, but patients are unlikely to adhere to exercise training interventions without demonstrable improvements in their symptoms. Symptom scores are a frequently assessed outcome in studies investigating the effects of an exercise intervention patients with asthma. Whilst any improvements demonstrated are likely to be multifactorial, a better understanding of the mechanism through which they occur is fundamental to the wider incorporation of exercise intervention into asthma management, both in children and adults.

A study of an exercise intervention in overweight and obese children with asthma demonstrated improvement in symptom scores [34]. However, this was associated with a reduction in weight, so it is difficult to tease apart whether this was a result of reduced asthma-driven inflammation or because of reduction in BMI.

Improvements in quality of life scores are one of the most widely studied areas in which exercise can exert a positive effect in asthma,

Exercise training programmes in children with asthma have shown significant improvements in quality of life $[34,37]$. These were associated with improvements in BMI in obese children and asthma-related inflammation [37].

Exercise training has shown a beneficial effect on the symptoms of anxiety and depression in meta-analyses, with greatest effects on depression [58]. A Cochrane review of exercise training in asthma concluded that exercise training-related improvements in quality of life may result in improved psychosocial well-being [59] Anxiety and depression are prevalent in asthma, particularly within difficult and severe asthma [60], and depression is related to higher levels of hospital admission, corticosteroid use [61] and poorer quality of life [62].

Studies in children to date have not specifically investigated psychosocial morbidity but, as discussed above, there have been improvements in quality of life scores, which contain some questions relevant to psychosocial morbidity.

\section{Effects of exercise on symptom scores, quality of life and psychosocial morbidity in} adults with asthma

Studies in adults have demonstrated improvements in symptom scores with exercise interventions, as assessed by the ACQ or Asthma Control Test scores [11, 42, 49, 51, 52], with a recent Cochrane review suggesting that physical training improves asthma control [52].

The majority of studies investigating the role for exercise training in asthma report on quality of life as an outcome measure $[11,42,63]$, with a variety of exercise training programmes including constant work rate and high-intensity interval training. Reviews support the positive effect of exercise on quality of life $[8,59,64,65]$.

Exercise training programmes in asthma patients have demonstrated significant improvements in anxiety and depression levels, with improvements in health-related quality of life and symptom scores [43 45, 47].

Potential mechanisms to explain the effect of exercise on symptom scores, quality of life and psychosocial morbidity in asthma

Whether symptom scores reduce through an improvement in disease related inflammation though a more generalised improvement in fitness has not been assessed, and this remains key to targeting of exercise interventions in asthma.

It may be impossible to tease out whether the improvements in quality of life are because of improved symptom control or other changes associated with exercise training. However, it seems that quality of life improvements are among the first [66] and sometimes only significant improvement demonstrated with exercise training [49].

The mechanisms behind improvements in psychosocial morbidity in both children and adults also remain unclear and are most likely multifactorial, with contributions from an increase in post-exercise dopamine expression and improved symptom control. At a mechanistic level, molecular and biochemical changes induced by exercise may underpin improvements in psychological status, with oxidative stress implicated in depression and increased levels of antioxidants seen following antidepressant treatment [67]. A mouse model of asthma has demonstrated that exercise training decreased OVA-induced depression and anxiety 
behaviours demonstrated by the mice, as well as increasing antioxidant levels in the lung and hippocampus [68], with the authors suggesting a link between the two.

\section{Exercise training and the obese asthma phenotype}

The obese asthma phenotype is emerging as a distinct group of patients that demonstrate poorer asthma control, reduced treatment responsiveness, and increased asthma severity and exacerbation rates [69].

\section{Exercise interventions in obese asthma in children}

Exercise interventions in obese and overweight children with asthma have demonstrated a reduction in BMI and improvements in maximal fitness as assessed by maximal oxygen uptake [40], lung function and quality of life [34]. Given the associated weight loss, it is difficult to distinguish whether these improvements are weight related or asthma related. Inflammatory markers have not been assessed in conjunction with this.

\section{Exercise interventions in obese asthma in adults}

There are some small studies to support the role for exercise training in the adult obese asthma group, with pulmonary rehabilitation demonstrating a reduction in BMI in obese patients, and an improvement in fat-free mass index in both overweight and obese patients, with associated improvements in Asthma Control Test, dyspnoea perception and admission rates [9]. Similarly, a pilot of pulmonary rehabilitation, with exercise in the form of high-intensity intermittent training in obese asthma patients prior to bariatric surgery, demonstrated improved asthma control at surgery when compared to controls [48]. In another group, weight loss of $>5 \%$ in combination with exercise demonstrated improved symptom scores and a reduction in dynamic hyperinflation [70]. A year-long interdisciplinary intervention that included exercise training in obese asthma patients showed a reduction in asthma severity, improved lung function and increased expression of anti-inflammatory adipokines when compared to controls, with adiponectin identified as an independent factor for the improvement of lung function in both groups [71]. A randomised controlled trial of exercise training in in obese adults with asthma, investigating weight loss versus weight loss and exercise intervention, demonstrated that the exercise group achieved increased improvement in symptom scores, weight loss, aerobic capacity, lung function and airway and systemic inflammation [11]. The same study reported improvements in daily step counts in the exercise group, and in sleep latency and efficiency, and a reduced risk of developing obstructive sleep apnoea [47]. Interestingly, a weight loss and exercise intervention demonstrated improvement in symptom scores in addition to a reduction in neutrophilic inflammation within the airways, that in women was related to a reduction in gynoid adipose tissue, whereas in males, reduced neutrophilic inflammation was associated with a reduction in dietary saturated fat [46]. In this study, there was also a reduction in eosinophilic airway inflammation which was only associated with exercise training [46], suggesting that modification of inflammation through exercise is complex and potentially driven through a number of mechanisms.

\section{Potential mechanisms to explain the effect of exercise intervention in obese asthma}

The mechanistic link between asthma and obesity remains to be clarified, and there is some question of "chicken and egg"; does the chronic inflammation known to be associated with obesity drive the development of asthma, or is the shortness of breath associated with asthma a driver for reduced levels of activity resulting in increased weight gain and obesity? It seems that within obese asthma there are, as within asthma as a whole, more than one endotype of disease. A recent review suggested that the obesity associated with Th2 high early-onset obese asthma was a consequence of the asthma whereas in Th2 low, later-onset disease, the asthma was more likely a consequence of obesity [72]. These interactions are summarised in figure 1. Either way, it seems that exercise training should be beneficial in this group of patients. Regardless of whether the improvement in inflammatory parameters demonstrated in these studies were a result of reduced BMI and therefore obesity associated inflammatory drive, or a reduction in asthma-driven systemic inflammation, there appears to be a disease-modifying role for exercise in the obese asthma group.

\section{Barriers to exercise in asthma}

The first step to design and implement an exercise training programme for asthma patients is to understand what prevents patients with asthma from exercising, despite recommendations in guidelines to do so. Intensity of physical activity has been shown to be associated with increased peak expiratory flow, although causation could not be determined in this cross-sectional study [73], yet even patients with relatively mild disease have been shown to avoid physical activity because they are concerned about triggering asthma symptoms [74]. Asthma severity as assessed by forced expiratory volume in $1 \mathrm{~s}$ and methacholine challenge were not predictive of maximal oxygen uptake as a marker of aerobic fitness [74], hence disease burden alone cannot explain reluctance to engage in physical activity. 


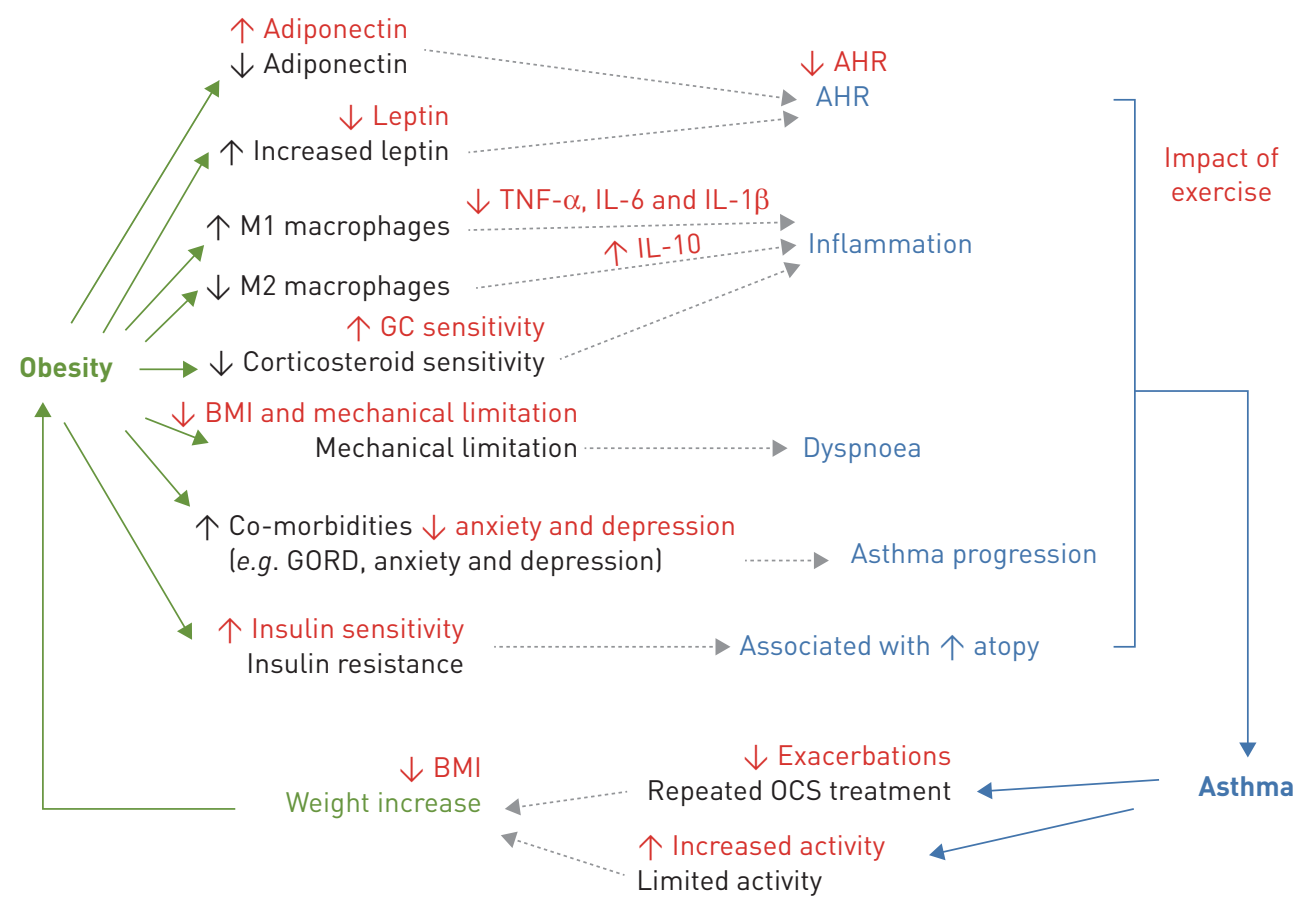

FIGURE 1 The interaction between asthma and obesity. AHR: airway hyperresponsiveness; OCS: oral corticosteroid; BMI: body mass index; GORD: gastro-oesophageal reflux disease; GC: glucocorticoid; TNF: tumour necrosis factor; IL: interleukin.

\section{Barriers to exercise in children with asthma}

Relatively few studies have investigated the barriers and facilitators to exercise and physical activity in asthma. The majority of these are in adolescents, partly because asthma tends to affect younger populations at a time when they should be establishing healthy lifestyles, and therefore it is a critical intervention point for encouraging long-term adoption of physical activity. Whilst a cross-sectional review suggests exercise is viewed by parents as beneficial in childhood asthma, with $97 \%$ of mothers agreeing, $37 \%$ of the same group admitted to imposing restrictions on physical activity with associations with mother's anxiety levels and severity of disease. Interestingly, the restrictive behaviour displayed by some of the mothers were not associated with lower levels of physical activity in their children [75]. Similarly, a study of elementary school teachers demonstrated few were aware that students with asthma need not avoid exercise [76]. Part of parental concern appears to be driven by worry regarding lack of symptom perception in children and lack of trust in school asthma management [77]. Other barriers have also been identified that prevent this group of patients in engaging with physical activity: fear of exacerbating symptoms, with patients with more severe disease more likely to view exercise as detrimental [78], and children's poor adherence to treatment also identified as barriers [77].

\section{Barriers to exercise in adults with asthma}

Adult studies similarly suggest that healthy participants and asthma patients think exercise is beneficial [79]. Fear of exacerbating symptom was also a common theme amongst adults [79]. Obesity and musculoskeletal problems, conditions that are common in asthma and exacerbated by oral steroid therapy, were also listed as a reason for not exercising, as were extreme weather conditions [79]. A lack of time is more likely to be reported as a barrier in younger patients [79]. Facilitators included the desire to be healthy and having encouragement from a motivated companion or physician, with lifestyle activities more acceptable to patients as a way to increase their physical activity levels [79]. In terms of intrinsic characteristics, patients with less asthma knowledge, lower self-efficacy and more negative attitudes towards asthma were more likely to view exercise negatively [79], also listed as a reason for not exercising, as were extreme weather conditions [79].

\section{Summary}

In summary, the evidence reviewed here supports the role of exercise in delivering wide-ranging health benefits in asthma, in terms of symptom perception, modification of mood, weight management and reduction in inflammation. Exercise could potentially play a key role as a therapeutic strategy in patients' care. Exercise training has the potential to effect improvements in many phenotypes and endotypes of 
disease, and we suggest it may be of benefit as part of a repertoire of treatments offered by difficult asthma services, along with other allied healthcare. Engagement with exercise training could be part of the optimisation process prior to biological treatment, with the potential to augment responses to these expensive therapies. We would suggest expanding guideline recommendations regarding exercise to include completion of a prescribed exercise training programme as part of routine management of asthma, with exercise viewed across all severities and endotypes as a standard "treatment" in the way that pulmonary rehabilitation has been incorporated into routine COPD care. Before this can happen, further mechanistic work is required to better elucidate the mechanisms behind the anti-inflammatory effect of exercise in asthma, to allow clarification of the optimal disease-modulating training programme. With regards to the anti-inflammatory benefit, the mechanism through which this is affected needs greater clarification, with a focus on the role of redox regulation. The mechanism of impact on symptom control and quality of life is of most relevance in relation to the anti-inflammatory story: are patients feeling better due to a reduction in disease associated inflammation as opposed to the well-known positive effects of exercise in general? If the psychosocial benefit of exercise is also mediated through a reduction in inflammation, then this has the potential to identify new strategies and medication targets for treating anxiety and depression. In obese asthma, it would be useful to determine whether exercise impacts its effect on the chicken or the egg; on asthma-driven inflammation or inflammation driven by adipose tissue.

Furthermore, given the effects of exercise training interventions on symptom control and fitness appear not to be sustained [39], patients need to adopt exercise as a lifestyle measure rather than a short-term intervention, and this requires improved understanding of the barriers and facilitators to exercise in asthma. Better understanding of these barriers will allow development of patient-centred training programmes to maximise uptake. A definitive study is needed to confirm the benefit of a defined exercise intervention for symptom control and reduction of inflammation in patients with asthma, with mechanistic investigation embedded within this efficacy study. Greater mechanistic understanding may help tailor exercise-based treatments to disease phenotypes, with huge health economic benefits. In the longer term, improved understanding of the immunomodulatory role of exercise in asthma may offer new opportunity to identify future drug targets that lead to improvements in airway inflammation, linking for the first time the benefits of exercise to the development of new pharmacological therapies.

Acknowledgements: The authors would like to thank Alastair Watson (Clinical and Experimental Sciences, University of Southampton, Faculty of Medicine, Southampton, UK) for his help in the preparation of the figure for this review.

Conflict of interest: A.T. Freeman has nothing to disclose. K.J. Staples reports grants from GSK Biologicals and AstraZeneca, outside the submitted work. T.M.A. Wilkinson reports personal fees and other from MMH, grants and personal fees from GSK, AstraZeneca and Synairgen, and personal fees from Boehringer Ingelheim, outside the submitted work.

\section{References}

Asher I, Pearce N. Global burden of asthma among children. Int J Tuberc Lung Dis 2014; 18: 1269-1278.

2 Murray CJ, Vos T, Lozano R, et al. Disability-adjusted life years (DALYs) for 291 diseases and injuries in 21 regions, 1990-2010: a systematic analysis for the Global Burden of Disease Study 2010. Lancet 2012; 380: 2197-2223.

3 Mallol J, Crane J, von Mutius E, et al. The International Study of Asthma and Allergies in Childhood (ISAAC) phase three: a global synthesis. Allergol Immunopathol (Madr) 2013; 41: 73-85.

4 O'Neill S, Sweeney J, Patterson CC, et al. The cost of treating severe refractory asthma in the UK: an economic analysis from the British Thoracic Society Difficult Asthma Registry. Thorax 2015; 70: 376-378.

5 Cruz AA, Stelmach R, Ponte EV. Asthma prevalence and severity in low-resource communities. Curr Opin Allergy Clin Immunol 2017; 17: 188-193.

6 Vijverberg SJH, Brinkman P, Rutjes NWP, et al. Precision medicine in severe pediatric asthma: opportunities and challenges. Curr Opin Pulm Med 2020; 26: 77-83.

7 British Thoracic Society. BTS/SIGN British Guideline on the Management of Asthma. brit-thoracic.org.uk/qualityimprovement/guidelines/asthma Date last updated: July 2019.

8 Avallone KM, McLeish AC. Asthma and aerobic exercise: a review of the empirical literature. J Asthma 2013; 50: $109-116$.

9 Candemir I, Ergun P, Kaymaz D. Efficacy of a multidisciplinary pulmonary rehabilitation outpatient program on exacerbations in overweight and obese patients with asthma. Wien Klin Wochenschr 2017; 129: 655-664.

10 Sahin H, Naz I. Comparing the effect of pulmonary rehabilitation in patients with uncontrolled and partially controlled asthma. J Asthma 2018: 1-8.

11 Freitas PD, Ferreira PG, Silva AG, et al. The role of exercise in a weight-loss program on clinical control in obese adults with asthma. A randomized controlled trial. Am J Respir Crit Care Med 2017; 195: 32-42.

12 Franca-Pinto A, Mendes FA, de Carvalho-Pinto RM, et al. Aerobic training decreases bronchial hyperresponsiveness and systemic inflammation in patients with moderate or severe asthma: a randomised controlled trial. Thorax 2015; 70: 732-739.

13 Nieman DC. Special feature for the Olympics: effects of exercise on the immune system: exercise effects on systemic immunity. Immunol Cell Biol 2000; 78: 496-501.

14 Walsh NP, Oliver SJ. Exercise, immune function and respiratory infection: an update on the influence of training and environmental stress. Immunol Cell Biol 2016; 94: 132-139. 
15 Global Initiative for Asthma. Global Strategy for Asthma Management and Prevention. 2018. https://ginasthma. org/wp-content/uploads/2018/04/wms-GINA-2018-report-tracked_v1.3.pdf

16 Jiang L, Diaz PT, Best TM, et al. Molecular characterization of redox mechanisms in allergic asthma. Ann Allergy Asthma Immunol 2014; 113: 137-142.

17 Onur E, Kabaroglu C, Gunay O, et al. The beneficial effects of physical exercise on antioxidant status in asthmatic children. Allergol Immunopathol (Madr) 2011; 39: 90-95.

18 Haldar P, Pavord ID, Shaw DE, et al. Cluster analysis and clinical asthma phenotypes. Am J Respir Crit Care Med 2008; 178: 218-224.

19 Drick N, Seeliger B, Welte T, et al. Anti-IL-5 therapy in patients with severe eosinophilic asthma - clinical efficacy and possible criteria for treatment response. BMC Pulm Med 2018; 18: 119.

20 Snelder SM, Weersink EJM, Braunstahl GJ. 4-month omalizumab efficacy outcomes for severe allergic asthma: the Dutch National Omalizumab in Asthma Registry. Allergy Asthma Clin Immunol 2017; 13: 34.

21 Silva RA, Vieira RP, Duarte AC, et al. Aerobic training reverses airway inflammation and remodelling in an asthma murine model. Eur Respir J 2010; 35: 994-1002.

22 Holgate ST. Pathogenesis of asthma. Clin Exp Allergy 2008; 38: 872-897.

23 Pastva A, Estell K, Schoeb TR, et al. Aerobic exercise attenuates airway inflammatory responses in a mouse model of atopic asthma. J Immunol 2004; 172: 4520-4526.

24 Pastva A, Estell K, Schoeb TR, et al. RU486 blocks the anti-inflammatory effects of exercise in a murine model of allergen-induced pulmonary inflammation. Brain Behav Immun 2005; 19: 413-422.

25 Vieira RP, Claudino RC, Duarte AC, et al. Aerobic exercise decreases chronic allergic lung inflammation and airway remodeling in mice. Am J Respir Crit Care Med 2007; 176: 871-877.

26 Hewitt M, Creel A, Estell K, et al. Acute exercise decreases airway inflammation, but not responsiveness, in an allergic asthma model. Am J Respir Cell Mol Biol 2009; 40: 83-89.

27 Lowder T, Dugger K, Deshane J, et al. Repeated bouts of aerobic exercise enhance regulatory $\mathrm{T}$ cell responses in a murine asthma model. Brain Behav Immun 2010; 24: 153-159.

28 Vieira RP, Toledo AC, Ferreira SC, et al. Airway epithelium mediates the anti-inflammatory effects of exercise on asthma. Respir Physiol Neurobiol 2011; 175: 383-389.

29 Silva AC, Vieira RP, Nisiyama M, et al. Exercise inhibits allergic lung inflammation. Int J Sports Med 2012; 33: 402-409.

30 Dugger KJ, Chrisman T, Jones B, et al. Moderate aerobic exercise alters migration patterns of antigen specific T helper cells within an asthmatic lung. Brain Behav Immun 2013; 34: 67-78.

31 Bruggemann TR, Avila LC, Fortkamp B, et al. Effects of swimming on the inflammatory and redox response in a model of allergic asthma. Int I Sports Med 2015; 36: 579-584.

32 Alberca-Custodio RW, Greiffo FR, MacKenzie B, et al. Aerobic exercise reduces asthma phenotype by modulation of the leukotriene pathway. Front Immunol 2016; 7: 237.

33 Fernandes P, de Mendonca Oliveira L, Bruggemann TR, et al. Physical exercise induces immunoregulation of Treg, M2, and pDCs in a lung allergic inflammation model. Front Immunol 2019; 10: 854.

34 Willeboordse M, van de Kant KDG, Tan FE, et al. A multifactorial weight reduction programme for children with overweight and asthma: a randomized controlled trial. PLoS One 2016; 11: e0157158.

35 Silva RA, Almeida FM, Olivo CR, et al. Exercise reverses OVA-induced inhibition of glucocorticoid receptor and increases anti-inflammatory cytokines in asthma. Scand J Med Sci Sports 2016; 26: 82-92.

36 Counil F-P, Varray A, Matecki S, et al. Training of aerobic and anaerobic fitness in children with asthma. J Pediatr 2003; 142: 179-184.

37 Moreira A, Delgado L, Haahtela T, et al. Physical training does not increase allergic inflammation in asthmatic children. Eur Respir J 2008; 32: 1570-1575.

$38 \mathrm{Lu} \mathrm{KD}$, Cooper D, Haddad F, et al. Glucocorticoid receptor expression on circulating leukocytes in healthy and asthmatic adolescents in response to exercise. Pediatr Res 2017; 82: 261-271.

39 Abdelbasset WK, Alsubaie SF, Tantawy SA, et al. Evaluating pulmonary function, aerobic capacity, and pediatric quality of life following a 10-week aerobic exercise training in school-aged asthmatics: a randomized controlled trial. Patient Prefer Adherence 2018; 12: 1015-1023.

40 Lucas JA, Moonie S, Hogan MB, et al. Efficacy of an exercise intervention among children with comorbid asthma and obesity. Public Health 2018; 159: 123-128.

41 Winn C, McNarry M, Stratton G, et al. Effect of high-intensity exercise on aerobic performance and airway inflammation in asthma. Eur Respir J 2016; 48: Suppl. 60, OA4804.

42 Farid R, Azad FJ, Atri AE, et al. Effect of aerobic exercise training on pulmonary function and tolerance of activity in asthmatic patients. Iran J Allergy Asthma Immunol 2005; 4: 133-138.

43 Mendes FA, Goncalves RC, Nunes MP, et al. Effects of aerobic training on psychosocial morbidity and symptoms in patients with asthma: a randomized clinical trial. Chest 2010; 138: 331-337.

44 Mendes FA, Almeida FM, Cukier A, et al. Effects of aerobic training on airway inflammation in asthmatic patients. Med Sci Sports Exerc 2011; 43: 197-203.

45 Turner S, Eastwood P, Cook A, et al. Improvements in symptoms and quality of life following exercise training in older adults with moderate/severe persistent asthma. Respiration 2011; 81: 302-310.

46 Scott HA, Gibson PG, Garg ML, et al. Dietary restriction and exercise improve airway inflammation and clinical outcomes in overweight and obese asthma: a randomized trial. Clin Exp Allergy 2013; 43: 36-49.

47 Freitas PD, Silva AG, Ferreira PG ADAS, et al. Exercise improves physical activity and comorbidities in obese adults with asthma. Med Sci Sports Exerc 2018; 50: 1367-1376.

48 Turk Y, van Huisstede A, Hiemstra PS, et al. Pre-surgical pulmonary rehabilitation in asthma patients undergoing bariatric surgery. Obes Surg 2017; 27: 3055-3060.

49 Toennesen LL, Meteran H, Hostrup M, et al. Effects of exercise and diet in nonobese asthma patients: a randomized controlled trial. J Allergy Clin Immunol Pract 2017; 6: 803-811.

50 Prossegger J, Huber D, Grafetstatter C, et al. Winter exercise reduces allergic airway inflammation: a randomized controlled study. Int J Environ Res Public Health 2019; 16: 2040.

51 Saxer S, Schneider SR, Appenzeller P, et al. Asthma rehabilitation at high vs. low altitude: randomized parallel-group trial. BMC Pulm Med 2019; 19: 134. 
52 Jaakkola JJK, Aalto SAM, Hernberg S, et al. Regular exercise improves asthma control in adults: a randomized controlled trial. Sci Rep 2019; 9: 12088.

53 Bentzon AK, Loehde LW, Backer V, et al. The long-term effect of an exercise and diet intervention in asthma patients: a 1-year follow-up on a randomised controlled trial. ERJ Open Res 2019; 5: 00032-2019.

54 Del Giacco SR, Scorcu M, Argiolas F, et al. Exercise training, lymphocyte subsets and their cytokines production: experience of an Italian professional football team and their impact on allergy. Biomed Res Int 2014; 2014: 429248.

55 Pakhale S, Luks V, Burkett A, et al. Effect of physical training on airway inflammation in bronchial asthma: a systematic review. BMC Pulm Med 2013; 13: 38.

56 Stephenson ST, Brown LA, Helms MN, et al. Cysteine oxidation impairs systemic glucocorticoid responsiveness in children with difficult-to-treat asthma. J Allergy Clin Immunol 2015; 136: 454-61.

57 Cumpstey AF, Minnion M, Fernandez BO, et al. Pushing arterial-venous plasma biomarkers to new heights: a model for personalised redox metabolomics? Redox Biol 2019; 21: 101113.

58 Wegner M, Helmich I, Machado S, et al. Effects of exercise on anxiety and depression disorders: review of metaanalyses and neurobiological mechanisms. CNS Neurol Disord Drug Targets 2014; 13: 1002-1014.

59 Carson KV, Chandratilleke MG, Picot J, et al. Physical training for asthma. Cochrane Database Syst Rev 2013; 9: CD001116.

60 Azim A, Mistry H, Harvey MA, et al. Comorbidities in the difficult asthma clinic: results from WATCH. Eur Respir J 2018; 52: Suppl. 62, PA1087.

61 Kullowatz A, Kanniess F, Dahme B, et al. Association of depression and anxiety with health care use and quality of life in asthma patients. Respir Med 2007; 101: 638-644.

62 Mangold R, Salzman GA, Williams KB, et al. Factors associated with depressive symptoms in uncontrolled asthmatics. J Asthma 2018; 55: 555-560.

63 Dogra S, Kuk JL, Baker J, et al. Exercise is associated with improved asthma control in adults. Eur Respir J 2011; 37: 318-323.

64 Lang JE. The impact of exercise on asthma. Curr Opin Allergy Clin Immunol 2019; 19: 118-125.

65 Eichenberger PA, Diener SN, Kofmehl R, et al. Effects of exercise training on airway hyperreactivity in asthma: a systematic review and meta-analysis. Sports Med 2013; 43: 1157-1170.

66 Freeman AT, Geale R, Bali S, et al. P105 high intensity intermittent exercise training in poorly controlled asthma: preliminary clinical trial results. Thorax 2018; 73: A159.

67 Liu T, Zhong S, Liao X, et al. A meta-analysis of oxidative stress markers in depression. PLoS One 2015; 10: e0138904.

68 Mokhtari-Zaer A, Hosseini M, Boskabady MH. The effects of exercise on depressive- and anxiety-like behaviors as well as lung and hippocampus oxidative stress in ovalbumin-sensitized juvenile rats. Respir Physiol Neurobiol 2018; 248: 55-62.

69 Marko M, Pawliczak R. Obesity and asthma: risk, control and treatment. Postepy Dermatol Alergol 2018; 35: 563-571.

70 Grandi Silva A, Duarte Freitas P, Ferreira PG, et al. Effects of weight loss on dynamic hyperinflation in obese women asthmatics. J Appl Physiol 2019; 126: 413-421.

71 da Silva PL, de Mello MT, Cheik NC, et al. Interdisciplinary therapy improves biomarkers profile and lung function in asthmatic obese adolescents. Pediatr Pulmonol 2012; 47: 8-17.

72 Leiria LO, Martins MA, Saad MJ. Obesity and asthma: beyond T(H)2 inflammation. Metab Clin Exp 2015; 64: $172-181$.

73 Malkia E, Impivaara O. Intensity of physical activity and respiratory function in subjects with and without bronchial asthma. Scand J Med Sci Sports 1998; 8: 27-32.

74 Garfinkel SK, Kesten S, Chapman KR, et al. Physiologic and nonphysiologic determinants of aerobic fitness in mild to moderate asthma. Am Rev Respir Dis 1992; 145: 741-745.

75 Dantas FM, Correia MA Jr, Silva AR, et al. Mothers impose physical activity restrictions on their asthmatic children and adolescents: an analytical cross-sectional study. BMC Public Health 2014; 14: 287.

76 Bruzzese JM, Unikel LH, Evans D, et al. Asthma knowledge and asthma management behavior in urban elementary school teachers. J Asthma 2010; 47: 185-191.

77 Kornblit A, Cain A, Bauman LJ, et al. Parental perspectives of barriers to physical activity in urban schoolchildren with asthma. Acad Pediatr 2018; 18: 310-316.

78 Winn CON, Mackintosh KA, Eddolls WTB, et al. Perceptions of asthma and exercise in adolescents with and without asthma. J Asthma 2018; 55: 868-876.

79 Mancuso CA, Sayles W, Robbins L, et al. Barriers and facilitators to healthy physical activity in asthma patients. J Asthma 2006; 43: 137-143. 